\title{
Fluid Attenuated Inversion Recovery
}

National Cancer Institute

\section{Source}

National Cancer Institute. Fluid Attenuated Inversion Recovery. NCI Thesaurus. Code C82392.

A magnetic resonance imaging (MRI) pulse sequence that uses an inversion recovery technique to null fluids present in the imaging area, improving clarity of the object of interest. 